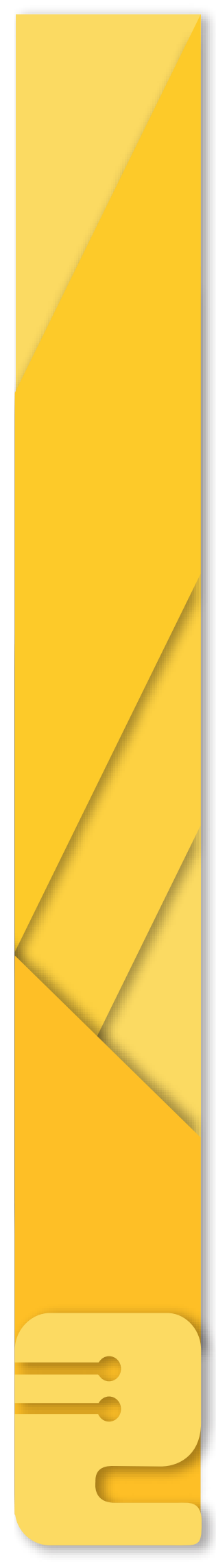

\title{
MODELOS MATEMÁTICOS Y ESTOCÁSTICOS PARA CONTROL DEL INVENTARIO EN BANCOS DE SANGRE: REVISIÓN DE LA LITERATURA $^{1}$
}

\author{
MATHEMATICAL AND STOCHASTIC MODELS FOR INVENTORY CONTROL IN BLOOD BANKS: \\ LITERATURE REVIEW \\ MODELOS MATEMÁTICOS E ESTOCÁSTICOS PARA CONTROLE DO INVENTÁRIO EM \\ BANCOS DE SANGUE: REVISÃO DA LITERATURA \\ Jairo Arboleda Zúñiga²
}

Fecha de recibido: Febrero 15 de 2017 | Fecha de aprobado: Abril 05 de 2017

\section{Resumen}

En este artículo se presenta una revisión de la literatura de las diversas metodologías utilizadas en la construcción de algunos de los más importantes modelos matemáticos y estocásticos que permiten el control del inventario en bancos de sangre, producto perecedero de alto impacto en la conservación de la vida de los seres humanos. Esta revisión se realizó utilizando setenta referencias bibliográficas de las investigaciones más destacadas desde la década de los 70 hasta la fecha, en la que se incluye los diversos comportamientos de la demanda y la donación, la sustitución de los diversos tipos de sangre, la condición multiproducto de los ítems, el deterioro y obsolescencia de los productos sanguíneos, las penalidades por faltantes y las diversas políticas de pedidos. Asimismo, se presentan los modelos más destacados para integrar la cadena de suministro, definida como la mejor estrategia para la optimización de tan vital recurso; al final, se destacan las brechas no cubiertas en este campo de conocimiento, que constituyen en desafíos para futuras investigaciones.

Palabras clave: modelos matemáticos - estocásticos, control de inventarios sanguíneos, bancos de sangre.

1 Artículo de revisión.

2 Ingeniero Industrial, Universidad del Valle (1985), Magister en Ingeniería Industrial, énfasis en Logística y Producción, Universidad del Valle (2013), Diplomado en Supply Chain Management, IRCC-Florida-USA. (1999). 24 años de experiencia laboral en funciones de gestión logística, gestión de la calidad y mejoramiento continuo en empresas multinacionales, pymes de la región e instituciones públicas. Es docente de la Facultad de Ingeniería de la Universidad Santiago de Cali e investigador del Grupo - Ideas (Competitividad y Productividad) categorizado "B" por Colciencias. Universidad Santiago de Cali - Colombia. Correo electrónico: jarboledaz@usc.edu.co 


\section{Abstract}

This article presents a literature review of the various methodologies used in the construction of some of the most important mathematical and stochastic models that allow the control of inventory in blood banks -perishable product of high impact in the conservation of life of human beings-. This review was carried out using seventy bibliographical references of the most outstanding investigations from the 70's to date, in which we included the various behaviors of demand and donation, substitution of various types of blood, multiproduct condition of the items, the deterioration and obsolescence of the blood products, the penalties for missing items and the diverse policies that regulate orders. Also, the most prominent models are presented to integrate the supply chain, defined as the best strategy for the optimization of this vital resource. In the end, the gaps not covered in this field of knowledge are highlighted, which constitutes a challenge for future research.

Key Words: mathematical-Estochastic Models, Blood Inventory Control, Blood Bank.

\section{Resumo}

Neste artigo apresenta-se uma revisão da literatura das diversas metodologias utilizadas na construção de alguns dos mais importantes modelos matemáticos e estocásticos que permitem o controle do inventário nos bancos de sangue, produto perecível de alto impacto na conservação da vida dos seres humanos. Esta revisão realizou-se utilizando setenta referências bibliográficas das investigações mais destacadas desde a década dos 70 até a data, na que se inclui os diversos comportamentos da demanda e a doação, a substituição dos diversos tipos de sangue, a condição multiproducto dos itens, a deterioração e obsolescencia dos produtos sanguíneos, as penalidades por faltantes e as diversas políticas de pedidos. Assim mesmo, apresentam-se os modelos mais destacados para integrar a corrente de fornecimento, definida como a melhor estratégia para a otimização de tão vital recurso; ao final, destacam-se as brechas não cobertas neste campo de conhecimento, que constituem em desafios para futuras investigações.

Palavras-chave: modelos matemáticos - estocásticos, controle de inventários sanguíneos, bancos de sangue. 


\section{INTRODUCCIÓN}

Un tema clave en la gestión de inventarios es el control de ítems perecederos (Vidal, 2010) ya que pueden llegar a un alto grado de obsolescencia en tiempos relativamente cortos; lo "perecedero" se refiere al deterioro de las unidades del inventario de un producto. Dentro de la gran familia de productos perecederos uno de los casos estudiados ha sido el abastecimiento de sangre debido a la complejidad en su manejo y por ser un producto vital para la sobrevivencia humana.

La sangre es un tejido vivo, es un sistema de conexión y transporte que hace posible el funcionamiento normal del organismo humano por permitir llevar el oxígeno, los nutrientes y químicos a todas las partes del cuerpo humano y contribuir a la expulsión de los desechos orgánicos (Kaushansky et al, 1995). Existen cuatro tipos básicos de células sanguíneas o componentes sanguíneos que se encuentran circulando en la sangre: los eritrocitos o glóbulos rojos, los leucocitos o glóbulos blancos, las plaquetas y el plasma sanguíneo (Williams, 2000).

Algunas características de la sangre como inventario, son:

- Inventario perecedero de vida fija: 6 horas (glóbulos blancos) / 5 días (plaquetas) / 35 días (sangre entera) / 42 días (glóbulos rojos) / 1 año (plasma).

- Inventario multiproducto: la sangre está clasificada en ocho tipos y varios componentes y cada uno tiene un uso diferente en el tratamiento médico de pacientes.

- Sustitución de componentes por tipo de sangre (compatibilidad).

- No es pertinente mencionar el asunto de los costos de mantener y adquirir el inventario por el sentido altruista de la donación y el marco legal que garantiza el suministro de sangre a todos los demandantes.

- En el $65 \%$ y $70 \%$ de los servicios hospitalarios no se conoce el tipo de sangre demandada.

- La oferta o abastecimiento de sangre es aleatorio y con sentido altruista.

- Desbalance entre la demanda (sistema Pull) y la recolección (sistema push).

Por las anteriores características anotadas, el modelamiento de inventarios en un banco de sangre implica cierta complejidad; además, en el proceso de almacenamiento, el inventario tiene que ser identificado por un "vector de estado" cuya dimensión es igual a la vida útil de la sangre; esta gran dimensión crea serios problemas computacionales, lo cual dificulta obtener una respuesta óptima; es por esto, que el desarrollo previo de modelos para productos perecederos no puede ser usado para obtener respuestas exactas en el caso de los inventarios sanguíneos.

La investigación de operaciones ha contribuido en la solución de muchos de los problemas de administración de un banco de sangre, especialmente en el nivel táctico (determinación de los niveles de inventarios, niveles de recolección, políticas de recolección y almacenamiento) y nivel operativo (programación y coordinación de: ordenes, proceso, recolección y almacenamiento). El banco de sangre de un centro hospitalario se puede considerar como una localidad de inventarios; que depende, entre otros factores, del número de donantes que hacen su aporte, de la vida útil de los componentes sanguíneos y del comportamiento de la demanda.

El objetivo propuesto en este artículo es presentar por medio de una revisión de la literatura científica obtenida de las más reconocidas fuentes bibliográficas contenidas en las bases de datos (Science Direct, Proquest, EBSCO, Scopus, Engineering Information, etc.), las distintas concepciones metodológicas tenidas en cuenta en la formulación de algunos importantes modelos matemáticos y estocásticos que han contribuido a encontrar soluciones para la administración de los bancos de sangre en instituciones hospitalarias. La revisión de la literatura se presenta en 5 secciones: (1) modelos matemáticos de inventarios para administración de bancos de sangre, (2) modelos estocásticos de inventarios para administración de bancos de sangre, (3) modelos que integran la cadena de abastecimiento, (4) investigaciones recientes, y, (5) investigaciones futuras.

\section{REVISIÓN DE LA LITERATURA}

\section{Modelos matemáticos de inventarios para administración de bancos de sangre}

En el desarrollo de los modelos de inventarios para bancos de sangre se pueden identificar 2 tipos clásicos de modelos definidos a partir del comportamiento de la demanda: 
- Modelo matemático (demanda regular o determinística).

- Modelo estocástico (demanda irregular o probabilística).

La utilización de los modelos de inventarios para administrar la distribución y recolección de sangre inició con los trabajos de Van Zyl (1964) y continuaron en la década de 1970 y en los primeros años de 1980 con Nahmias (1982) y Prastacos (1984), quienes desarrollan una visión de la teoría y práctica de la administración de inventarios de sangre. Prastacos (1984) recopila de los últimos 15 años una serie de contribuciones metodológicas que la Investigación de Operaciones había realizado en el área de teoría de inventarios, aplicada a productos o sistema de salud. Estas contribuciones incluyen el desarrollo de técnicas que permiten desarrollar políticas de pedido exacto y aproximado de un sistema de inventario, el análisis de sistemas multiproductos y varias formas de programación de la distribución.

Fano y Longres (1997) ensayaron un modelo estimativo con el objetivo de calcular el inventario mínimo de glóbulos rojos y plasma, disponible en el Servicio de Hemoterapia del Instituto Superior de Medicina Militar “Dr. Luis Díaz Soto” de La Habana, para cubrir las necesidades diarias de todas las áreas clínicas y quirúrgicas, incluidas las de urgencias. El inventario mínimo de glóbulos rojos calculado garantiza la disponibilidad de sangre en cualquier situación de urgencia que se presente.

Jagannathan y Sen (1986) estudiaron específicamente el proceso de demanda para la prueba cruzada. Sus análisis asumieron demanda constante y determinística, pero esto no es muy realista.

Díaz Fernández, Brío González y González Torre (2001) consideran la posibilidad de modelización a través de criterios de programación por metas, que, usando los mismos algoritmos de resolución que la programación lineal, sustituyen las restricciones por metas flexibles, cuyas desviaciones se pretende minimizar, tratando de conseguir que las desviaciones que no son interesantes sean las menores posibles.

Kozan, (2001) plantea un modelo matemático para determinar la mejor política de despacho de unidades de glóbulos rojos a hospitales y laboratorios. El modelo desarrollado encuentra la política optima de distribución mientras minimiza el número de unidades obsoletas y mantiene un inventario de seguridad que satisface las políticas de desabastecimiento; el problema se formula como un modelo de programación lineal entera y lo resolvió usando "Gams" (General Algebraic Modeling System).

Ballou, (2004) en el artículo“American Red Cross: Blood Services", basado en un caso presentado en el Centro Regional de la Cruz Roja Americana en la región del norte de Ohio, presenta un análisis sobre el tiempo de vida de la sangre que se vence en un periodo y la escasez que se presenta en el siguiente periodo. En el artículo, se describen los principales factores que afectan las cantidades de sangre que se tienen en inventario, tales son:

- Demanda: número de unidades de sangre de cualquier tipo que requieren los bancos de sangre, puede ser conocida o aleatoria.

- Escasez: ocurre cuando la demanda excede del número de unidades de sangre en inventario.

- Tasa de escasez: la fracción a largo plazo (o porcentaje) de días en los cuales hay escasez.

- Tasa de escasez es igual al número de días en los que ocurren faltantes / número total de días.

- Caducidad: una unidad de sangre que es descartada por exceder su vida útil, 35 a 42 días para sangre entera.

- Tasa de caducidad: relación entre el número promedio de unidades con vida útil vencida de sangre y el número promedio de unidades recolectadas de sangre.

El nivel óptimo de inventario para un derivado sanguíneo específico se encuentra como una relación entre la tasa de escasez y la tasa de caducidad. El método general sugerido para el manejo de los inventarios en los centros regionales de sangre es de planeación de arriba hacia abajo, en el que se considera la planeación anual, mensual, semanal y diaria.

El modelo de control de inventarios de Jagannathan y Tarun (2005), utiliza parámetros de decisión que ayudan a conducir un análisis de sensibilidad asociado con los parámetros controlables del inventario de sangre.

Osorio, Brailsford y Smith (2014) proponen un modelo de programación lineal entera que contiene como objetivos la minimización del costo total y del número de donantes, sujeto a restricciones de capacidad, proporcionalidad de tipos de sangre y satisfacción de la demanda y en el que la solución del modelo se realizó con software libre, Open Solver 2.1. 


\section{MODELOS ESTOCÁSTICOS DE INVENTARIOS PARA ADMINISTRACIÓN DE BANCOS DE SANGRE}

Las primeras investigaciones a nivel de modelos estocásticos incluyeron simulación, regresión y análisis de cadenas de Markov para analizar diferentes políticas en un banco de sangre.

En lo referente a los modelos estocásticos desarrollados para el control de inventarios con demandas de sangre mediana y altamente variable se presentan las contribuciones más significativas, según las diferentes metodologías utilizadas:

\section{Simulación}

Los primeros investigadores que contribuyeron a esta área fueron Elston y Pickerel (1963) quienes usaron métodos estadísticos para analizar los efectos en un solo hospital, modificando la composición de la edad de los componentes y el tamaño del suministro de sangre. Ellos usaron la distribución binomial negativa para modelar la demanda.

Jennings (1973) fue el primero en expandir un modelo de inventarios de sangre a un plano regional en lugar de un solo hospital. Usando análisis de simulación, él revisa un sistema de varios hospitales con idénticos bancos de sangre tomando decisiones basadas en las mismas políticas.

Cohen y Pierskalla (1975) intentaron usar ecuaciones simples para encontrar los niveles óptimos de inventario; usando simulación, ellos determinaron un nivel aceptable de inventario basados en todas las variables significativas. Adicionalmente examinaron la sensibilidad de diferentes variables para el costo total y el inventario optimo utilizando los costos de obsolescencia y costo de agotados.

La siguiente contribución viene de Brodheim, Hirsch y Prastacos (1976) quienes estudiaron los datos operacionales para desarrollar relaciones entre la demanda y los niveles de inventario a una razón específica de agotamiento. Usaron regresiones y arreglos lineales y ecuaciones cuadráticas, logrando predicciones de alta exactitud en los niveles de inventario.

Abbot (1977), fue el primero en desarrollar un modelo incorporando varios tipos de sangre, mientras que todos los trabajos anteriores habían utilizado sangre agregada. Él incluyó la simulación de los tipos de sangre, de esta manera redujeron los agotados, representando un modelo más realista.

Sirelson y Brodheim (1991), crearon un modelo de inventario de plaquetas usando simulación para predecir los agotados y las tasas de obsolescencia basados en los niveles de demanda e inventario.

Brennan, J. E., et al. (1992), mejoran la programación de las donaciones estudiando la personalidad de los donantes y las instalaciones para administrar la recolección de sangre.

Pierskalla (2004), mejora el registro de donantes y monitoreo de la cadena de abastecimiento de bancos de sangre, involucrando un análisis de simulación y regresión en el modelado de los niveles de inventario en el banco de sangre de un hospital y los centros comunitarios de sangre.

Pereira (2005) plantea un modelo estocástico que simula la operación del banco de sangre de un centro médico con un numero finito de días, esto permitió que se analizaran diversos factores que influyen en las tasas de escasez y de obsolescencia, incluyendo la media y el coeficiente de variación (CVD) en las transfusiones diarias, concluyendo que este coeficiente es el mejor parámetro para determinar el desempeño de un banco de sangre.

\section{Cadenas de Markov y Programación Dinámica}

Pegels y Jelmert (1970) desarrollaron un modelo de cadena de Markov, gracias a la utilización de un estado absorbente de la cadena para analizar cómo los niveles de inventario y la edad promedio de sangre transfundida era afectada por las diversas políticas emitidas; dicho modelo requiere las probabilidades de transición de que una unidad de sangre pueda ser transfundida según su edad o vida útil.

Kolesar (1973) considera el anterior modelo como incompleto y poco confiable al puntualizar que las probabilidades de transición también son una función de otras variables como la razón de demanda y el nivel normal de inventario y no solo del tiempo de vida del componente.

Brodheim, Derman y Prastacos (1979) aplicaron cadenas de Markov para analizar el suministro de sangre y la demanda, sus modelos también requieren probabilidades de transición, y fueron fundamentales para la creación del Sistema Programado de 
Decisiones Sanguíneas, un modelo de computador que ayuda en la programación de entregas a los hospitales.

Fries y Nahmias (1975) aplicaron la programación dinámica, obteniendo conclusiones muy valiosas para el inventario perecedero en general: la cantidad óptima a pedir no es tan sensible a los cambios en el inventario más viejo como si lo es para el inventario más nuevo. Nahmias (1982), además concluyó que trabajando con inventarios que tienen una expectativa de tiempo de vida mayor que tres periodos es extremadamente difícil precisar resultados.

Cohen (1976) modeló la administración del inventario de sangre como un proceso estocástico usando la emisión de política FIFo, es cierto que se tuvo en cuenta la demanda, pero se asumió que todas las unidades asignadas son utilizadas, lo cual no es real debido a la prueba cruzada.

Liu y Lian (2001) desarrollan un modelo para ítems perecederos, de revisión continua con un proceso general de reabastecimiento de la demanda; usan la teoría regenerativa de Markov y construyen una función de costo cerrada donde se permiten los "backorders" u órdenes pendientes; los autores también concluyen que es improbable que la política optima de ordenamiento, pueda ser encontrada para modelos generales de inventarios perecederos con "lead time" positivos.

Kalpakam y Shanthi (2006), proponen un modelo para control de ítems perecederos con demanda tipo “Poisson", en el que las órdenes son colocadas solamente durante épocas de demanda, y utilizan los procesos semi-regenerativos para atacar el problema de incremento en la reposición de la demanda, cantidad variable de la orden y "lead time" positivo.

Las plaquetas son el componente sanguíneo más perecedero y tienen una demanda altamente variable e incierta; la demanda y obtención son periódicas; la Programación Dinámica de Markov (MDP), combinada con simulación, se aplicó en el banco de sangre de un hospital de Alemania (Haijema, Van der Wal, \& Van Dijk, 2007) y se demostró que disminuyendo la dimensión y aplicando el análisis combinatorio, se pueden definir reglas de reposición para mejorar el servicio.

\section{Dinámica de sistemas}

La dinámica de sistemas es básicamente una filosofía, una forma de observar las organizaciones y los sistemas; una metodología para el estudio de los sistemas complejos y la interacción entre las variables del sistema. Este concepto sistémico integra el conocimiento acerca del mundo real con el concepto de alimentación de estructuras causales que cambian a través del tiempo (Forrester, 1973).

Jennings (1973) encabeza el grupo de investigadores que consideran el banco de sangre como un sistema dinámico. El flujo de la sangre es similar al flujo de material de un sistema dinámico y con el uso de este enfoque es posible encontrar en la realidad, mejores resultados.

Figura 1. Modelo dinámico clásico de bancos de sangre

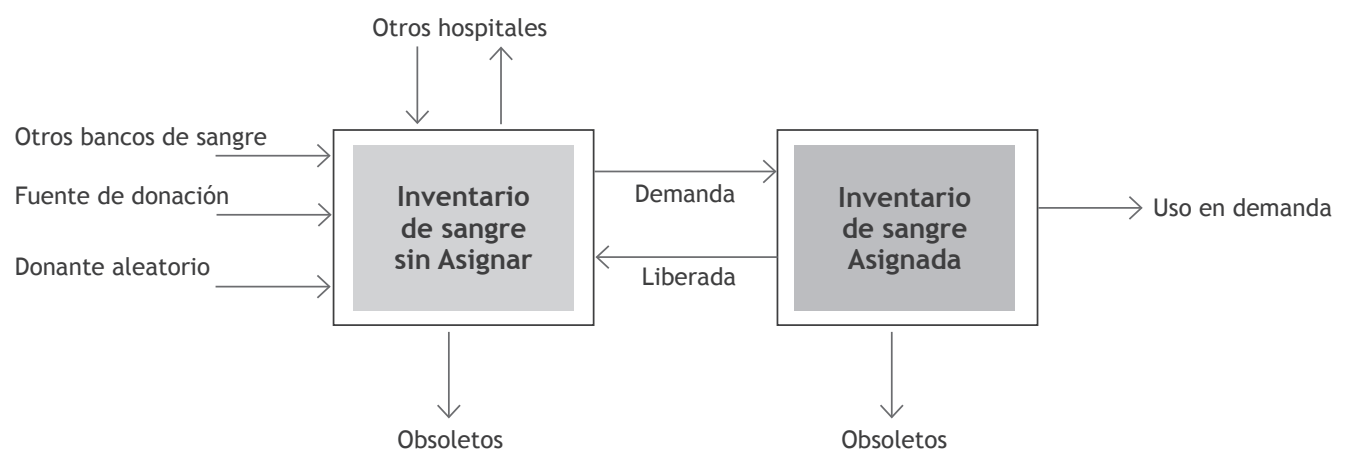

Fuente: adaptado de Jennings (1973).

En el proceso de modelamiento se usan dos importantes esquemas para conducir la dinámica de sistemas, uno está pensado en cómo las cantidades varían a través del tiempo y el otro en cómo se presenta la retroalimentación sustancial. Roberts, Andersen, Deal, Garet y Shaffer (1983) muestran lo anterior a través del siguiente gráfico: 
Figura 2. Diagrama causal para inventarios de sangre

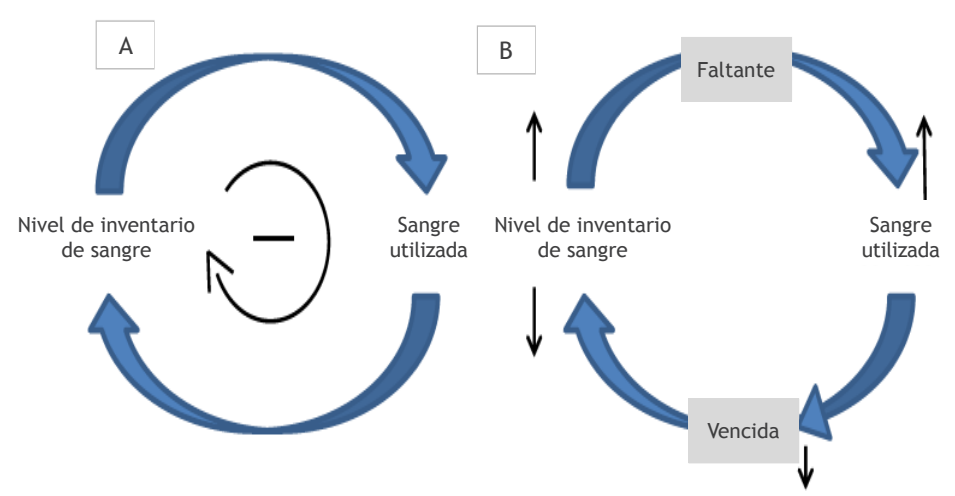

Fuente: adaptado de Roberts (1983).

Shoukath y Ramaswamy (1991) aplican técnicas estadísticas para confirmar la confiabilidad de las herramientas de dinámica de sistemas usadas en el modelado y análisis de la administración de un sistema de inventarios de sangre; utilizan medidas de desempeño numérico (NPM), hacen uso de series de tiempo y concluyen que la desviación estándar (DE) es una herramienta fundamental para realizar inferencias sobre el comportamiento y evaluación del sistema de inventario de sangre.

\section{Modelos de inventarios para integrar la cadena de abastecimiento de la sangre}

Vaughan (1988) en su tesis doctoral de la Universidad de lowa plantea un modelo para el suministro de sangre en el que describe la red del sistema de inventario de un hospital a través del análisis de estado de la cadena de Markov. Este modelo, luego fue extendido por niveles a un gran centro regional, dando la distribución de probabilidad del ingreso de donantes y las ordenes esperadas para los hospitales, lo que resolvió el envejecimiento promedio de las unidades a entregar, así como la probabilidad de desabastecimiento.

Chapman, Hyam y Hick (2004) sostienen que la única forma de lograr la mayor eficiencia del complejo sistema de inventario sanguíneo es mediante el trabajo unido de todos los componentes de la cadena de suministro.

La institución NBS (National Blood Services of England) en Inglaterra, utiliza proyecciones con base en la demanda planeada que regularmente espera y elabora estas proyecciones de demanda para el corto y mediano plazo. Los factores que influyen en la demanda son: la demografía poblacional, los avances en las técnicas médico-quirúrgicas y los procedimientos de diagnóstico y manejo traumático, además de las iniciativas para un mejor uso de los glóbulos rojos y demás derivados de la sangre.

A nivel latinoamericano se han venido realizando esfuerzos para consolidar la cadena de suministro de sangre a través de grandes centros regionales, a este respecto vale la pena resaltar el trabajo realizado por el Centro de Sangre de Concepción (Chile) que hoy en día es la única organización abastecedora de sangre que surte más de una veintena de establecimientos hospitalarios del servicio de salud del sur del país. El anterior modelo de abastecimiento de sangre ha sido replicado en otras regiones del país como Valparaíso, Santiago y Antofagasta; desde el año 2007, esta red cubre la necesidad de los hospitales del territorio nacional.

Actualmente, en un hospital del Reino Unido por medio del modelamiento de la cadena de suministros se ha implementado un sistema (Katsaliaki \& Brailsford, 2007) para mejorar los procedimientos, el control y salidas del inventario de sangre; la red es descompuesta en flujo de materiales y flujo de información; la simulación de eventos discretos es utilizada para mejorar la coordinación del sistema y determinar la política de ordenamiento, como guía para reducir los faltantes y el desperdicio.

A continuación, se presenta un cuadro que resume la apropiación de las diversas metodologías tenidas en cuenta en el desarrollo de los modelos matemáticos y estocásticos para control del inventario de componentes sanguíneos en la década comprendida entre 1997 y 2007, destacándose las aplicaciones de origen estocásticas $(68.3 \%)$ sobre las determinísticas 
(31.7\%) pero con una fuerte tendencia hacia la búsqueda de soluciones confiables al combinar métodos de aproximación con métodos exactos.

Tabla 1. Tendencia en el uso de las metodologías para control de inventarios sanguíneos entre año 1997 a 2007

\begin{tabular}{|l|c|}
\hline \multicolumn{1}{|c|}{ METODOLOGÍA } & $\begin{array}{c}\text { PORCENTAJE } \\
\text { DE ARTÍCULOS } \\
\text { PUBLICADOS }\end{array}$ \\
\hline $\begin{array}{l}\text { Simulación } \\
\text { Programación dinámica estocástica } \\
\text { / cadenas de Markov }\end{array}$ & $35.9 \%$ \\
\hline $\begin{array}{l}\text { Programación lineal entera, mixta y } \\
\text { programación por metas }\end{array}$ & $23.3 \%$ \\
\hline $\begin{array}{l}\text { Metaheurísticas, heurísticas y } \\
\text { dinámica de sistemas }\end{array}$ & $21.2 \%$ \\
\hline $\begin{array}{l}\text { Análisis estadístico } \\
\text { Modelos de colas }\end{array}$ & $6.1 \%$ \\
\hline
\end{tabular}

Fuente: adaptado de Belien y Forcé (2012).

\section{INVESTIGACIONES RECIENTES EN MODELOS PARA ADMINISTRAR BANCOS DE SANGRE}

En los últimos años, pocas investigaciones han sido conducidas para administrar sistemas de abastecimientos de sangre. En Estados Unidos, por ejemplo, ha decrecido el número de investigaciones en este campo, debido a los recortes en los fondos federales para tal fin. Hoy por hoy, los esfuerzos se han dirigido más hacia la garantía en el suministro de sangre en toda la red, el perfeccionamiento de los modelos de pronósticos de demanda, el mejoramiento en la rapidez de solución de los modelos de programación lineal utilizando diversos algoritmos de búsqueda y metaheurísticas. Los estudios más destacados en los últimos diez años, se sintetizan en la siguiente tabla:

Tabla 2. Recopilación de los modelos matemáticos y estocásticos destacados entre los años 2007 - 2017

\begin{tabular}{|c|c|c|}
\hline AUTORES & METODOLOGÍA UTILIZADA & OBSERVACIONES (RESULTADOS) \\
\hline Haijema et al (2007) & $\begin{array}{l}\text { Programación dinámica } \\
\text { estocástica combinada con } \\
\text { simulación }\end{array}$ & $\begin{array}{l}\text { Alcanzan la definición de una política óptima de pedidos } \\
\text { de plaquetas en un banco de sangre y demuestran como } \\
\text { el modelo de simulación usado para glóbulos rojos pue- } \\
\text { de ser acoplado a un nuevo algoritmo híbrido de opti- } \\
\text { mización metaheurística para obtener una solución muy } \\
\text { cercana al óptimo. }\end{array}$ \\
\hline $\begin{array}{l}\text { Kopach, Balcioglu y } \\
\text { Carter (2008) }\end{array}$ & $\begin{array}{l}\text { Modelo de colas con técnicas } \\
\text { multinivel cruzada }\end{array}$ & $\begin{array}{l}\text { El modelo fue aprobado en la red de hemocentros del } \\
\text { Canadá y ayudó a las transacciones entre: niveles múl- } \\
\text { tiples de demanda, niveles de servicio y costos, bajo el } \\
\text { objetivo tradicional de minimizar los agotados y obso- } \\
\text { letos. }\end{array}$ \\
\hline $\begin{array}{l}\text { Broyles, Cochran y } \\
\text { Montgomery (2010) }\end{array}$ & $\begin{array}{l}\text { Modelo de probabilidad de } \\
\text { cadenas de Markov }\end{array}$ & $\begin{array}{l}\text { Demuestran cómo la utilización de cadenas de Markov } \\
\text { en paralelo con modelo de pronósticos Arima permite } \\
\text { predecir la demanda estacional de pacientes con nece- } \\
\text { sidades de componentes sanguíneos. }\end{array}$ \\
\hline Belien y Forcé (2012) & $\begin{array}{l}\text { Modelos estadísticos sobre el } \\
\text { uso de modelos utilizados en la } \\
\text { administración de la cadena de } \\
\text { suministro de ítems sanguíneos }\end{array}$ & $\begin{array}{l}\text { Realizan una revisión de los modelos utilizados con ma- } \\
\text { yor éxito en los años } 2000 \text { y } 2010 \text {, las mayores problemá- } \\
\text { ticas a tratar y la toma de decisiones más importantes } \\
\text { que han sido consideradas en los modelos }\end{array}$ \\
\hline $\begin{array}{l}\text { Silva-Filho, Cezarino } \\
\text { y Salviano (2012) }\end{array}$ & $\begin{array}{l}\text { Modelo de pronósticos } \\
\text { autorregresivos- Sarima }\end{array}$ & $\begin{array}{l}\text { Sistema computarizado de soporte de decisiones para } \\
\text { el pronóstico de demanda que mejora la planeación y } \\
\text { control del inventario sanguíneo. }\end{array}$ \\
\hline Duan y Liao (2014) & $\begin{array}{l}\text { Simulación Optimizada (so) } \\
\text { con algoritmo de optimización } \\
\text { meta heurística, TA (Umbral de } \\
\text { aceptación) y HTS (búsqueda } \\
\text { tabú hibrida) }\end{array}$ & $\begin{array}{l}\text { En este modelo se maneja la restricción del tiempo } \\
\text { máximo de vida de los glóbulos rojos a través de un en- } \\
\text { foque de optimización bajo simulación y considerando } \\
\text { las compatibilidades de los diversos grupos de sangre } \\
\text { ABO. }\end{array}$ \\
\hline
\end{tabular}




\section{AUTORES}

METODOLOGÍA UTILIZADA

Silva-Filho, Cezarino, Modelo de pronósticos Box Silva y Salviano

(2013) Jenkins con factor estacional univariado -BJ-SARIMA

Modelo de programación lineal

Bellabdaoui, Bellis, entera donde el objetivo es Saadi y Benajiba (2014) minimizar el nivel de pérdida de inventario balanceando los diversos niveles de stock

\begin{tabular}{ll|} 
Hainema, R. (2014) & $\begin{array}{l}\text { Programación dinámica } \\
\text { estocástica }\end{array}$ \\
\hline
\end{tabular}

Gunpinar y Centeno (2015)

Modelo de programación entera para minimizar el costo total

Algoritmo genético para modelos de inventarios

Saracoglu, Topaloglu y Keskintur ( 2014)

multiproductos, multiperiódicos de revisión continua

Modelo genérico de simulación

Blake y Hardy (2014) para evaluar la red de abastecimiento de sangre

Kowalski, France, Whitehouse y Himawan (2014)

Estudio y análisis estadístico de las características de los donantes de sangre
Alizadeh, Eskandari, y Sajadifar (2014)

Modelo de programación lineal combinado con algoritmo de búsqueda del límite superior Modelo de simulación optimizado con múltiples restricciones de vida útil de glóbulos rojos

Ke-ming y Zu-jun (2015)

Simulación bajo diferentes escenarios según políticas de inventarios

\section{Modelo de análisis}

Duong, Wood y Wang (2015) multicriterio, incluyendo el inventario promedio, el fill rate o tasa de surtido y la tasa de variación de la orden
OBSERVACIONES (RESULTADOS)

Sistema de pronósticos de buena precisión (Sarima mejorado) que permite el monitoreo y control del inventario de sangre en todos los eslabones de la cadena de suministro.

Lograron obtener un inventario balanceado en los diferentes centros de abastamiento bajo un enfoque comercial de minimización de costos.

Investigan la reducción de costos que se logra por un pedido que dependiendo del tiempo de vida del componente, usando la filosofía fifo produce un alto valor agregado a la política de inventarios.

En la propuesta también se incluyeron modelos estocásticos y determinísticos para incluir el grado de variabilidad e incertidumbre de la demanda, los tipos de sangre, las pruebas y las transfusiones. Los resultados mostraron una disminución en $17.2 \%$ en el desperdicio y un $20.7 \%$ en el costo.

La solución de un modelo de programación lineal entera se redujo utilizando algoritmo genético, el cual permite encontrar soluciones a problemas de gran escala definiendo la trayectoria de búsqueda que permite obtener buenas soluciones de forma rápida.

Concluyen que un modelo genérico puede ser útil para los bancos de sangre regionales y sugiere utilizar un mínimo de 4 escenarios para los nodos de abastecimiento.

Definieron los cuatro factores fundamentales que influyen en la donación de sangre individual: síntomas de síncope, fobia a las agujas y a la sangre, evolución social y resultados médicos para ser donante aprobado.

Útil en el caso de demanda estocástica y lead time diferente de cero. Permite un análisis de sensibilidad confiable ante el cambio de varios parámetros.

Modelo que permite el uso de sangre según las compatibilidades sanguíneas de RH y tipos de sangre que minimiza los vencimientos y el desabastecimiento.

Introduce los métodos de transbordo de acuerdo a diversas variaciones de suministro y demanda, tiempo de vida de los componentes y enfatiza el uso de la política fIFo en los transbordos para reducir los agotados.

Consideraron la complejidad del inventario de sangre originada por los aspectos perecederos y la sustitución de productos y propusieron métricas para manejar estas dos condiciones. 


\begin{tabular}{|c|c|c|}
\hline AUTORES & METODOLOGÍA UTILIZADA & OBSERVACIONES (RESULTADOS) \\
\hline $\begin{array}{l}\text { Hosseinifard y Abbasi } \\
\text { (2016) }\end{array}$ & $\begin{array}{l}\text { Modelo de redes que utiliza } \\
\text { programación lineal entera } \\
\text { mixta con dos eslabones: uno } \\
\text { donde se incluye un banco } \\
\text { de sangre que recibe el } \\
\text { abastecimiento aleatorio de } \\
\text { los donantes, y que contienen } \\
\text { los hospitales que realizan las } \\
\text { transfusiones }\end{array}$ & $\begin{array}{l}\text { Demostraron que la centralización del inventario es un } \\
\text { factor clave en la cadena de suministro sanguínea y pue- } \\
\text { de incrementar la sostenibilidad y resiliencia en la red; } \\
\text { en los estudios se demostró que reduciendo de siete a } \\
\text { tres el número de hospitales que mantenían la sangre, } \\
\text { decrecía en } 40 \% \text { los agotados y en } 21 \% \text { los componentes } \\
\text { vencidos. }\end{array}$ \\
\hline $\begin{array}{l}\text { Lowalekara y Ravi } \\
\text { (2017) }\end{array}$ & $\begin{array}{l}\text { Modelo de aplicación } \\
\text { utilizando la filosofía "Teoría } \\
\text { de Restricciones - Toc" al } \\
\text { medio de los bancos de sangre }\end{array}$ & $\begin{array}{l}\text { Mostraron las relaciones existentes entre los altos des- } \\
\text { perdicios de sangre por vencimiento y obsolescencia, } \\
\text { alto nivel de inventario, escasa y errática captación de } \\
\text { sangre, altos costos de operación a través de un diagra- } \\
\text { ma de árbol de problemas para detectar la causa raizal. }\end{array}$ \\
\hline $\begin{array}{l}\text { Dillon, Oliveira y } \\
\text { Abbasi (2017) }\end{array}$ & $\begin{array}{l}\text { Modelo de programación } \\
\text { estocástica en dos etapas: } \\
\text { el tiempo de vida útil y la } \\
\text { demanda incierta }\end{array}$ & $\begin{array}{l}\text { Permitió definir la política óptima de revisión en la ges- } \\
\text { tión del inventario de glóbulos rojos enfocándose en la } \\
\text { minimización de los costos operativos, así como tam- } \\
\text { bién los desabastecimientos y el desperdicio debido a } \\
\text { los vencimientos. }\end{array}$ \\
\hline $\begin{array}{l}\text { Puranam, Novak, } \\
\text { Lucas y Fung ( 2017) }\end{array}$ & $\begin{array}{l}\text { Programación dinámica con } \\
\text { minimización multiperiódica } \\
\text { de los costos }\end{array}$ & $\begin{array}{l}\text { El modelo suministra una contribución teórica y admi- } \\
\text { nistrativa mediante la consideración de múltiples fuen- } \\
\text { tes de abastecimiento. }\end{array}$ \\
\hline
\end{tabular}

Fuente: elaboración propia.

\section{Investigaciones futuras}

A continuación se describen algunos aspectos no tratados o tratados hasta ahora con poca profundidad que se convierten en brechas del conocimiento en la temática tratada y constituyen grandes oportunidades para investigaciones futuras:

- La mayoría de estudios han sido direccionados al componente sanguíneo más utilizado en los procedimientos hospitalarios, los glóbulos rojos, y hay muy pocos estudios para los otros componentes: plaquetas, plasma y crio precipitados.

- Escasa atención han recibido los modelos de asignación de vehículos encargados de la recolección y distribución de la sangre: ruteo, asignación de inventario y programación de los múltiples productos sanguíneos desde los diferentes centros regionales de abastecimiento hematológicos de la ciudad a los diversos centros hospitalarios locales y regionales.

- Escasez de estudios que traten la asignación de costos de los productos sanguíneos y la colaboración inter-regional para reducción de costos y el mejoramiento del servicio.

- Muy poco material investigativo en lo referente al uso para rotulado y almacenamiento de productos sanguíneos a través de sistemas de identificación por radiofrecuencias que garanticen la trazabilidad en toda la cadena de abastecimiento.

- Faltan mayores esfuerzos para el diseño de sistemas de información que soporten las decisiones sobre gestión de inventarios de sangre de forma rápida y oportuna.

- Faltan estudios más confiables para analizar y medir el impacto de los tiempos de ciclo o "lead time" en los modelos de inventarios de sangre y la sustitución de productos según compatibilidad por tipo de sangre.

- Poco uso de herramientas estructuradas como el método Delphi, Proceso de Análisis JerárquiCO- AHP o panel de expertos para encontrar definiciones más precisas de las ponderaciones y penalizaciones en los modelos de programación de metas usadas para la gestión de inventarios sanguíneos.

\section{CONCLUSIONES}

En el presente artículo, se observó cronológicamente como fueron desarrollándose las diversas metodologías y técnicas utilizadas en el modelamiento matemático y estocástico para la administración de los sistemas de inventarios de sangre. Se encontró 
entre las técnicas más utilizadas las siguientes: programación dinámica, cadenas de Markov, simulación y análisis estadístico para las demandas con comportamiento aleatorio y programación lineal entera y programación lineal por metas para el comportamiento determinístico de la demanda. Así pues, se concluye que los modelos con mejores resultados en cuanto a satisfacción de la demanda, reducción de agotados y obsoletos son los que sustentan en programación lineal, pero con manejo aleatorio de las variables de decisión, una fusión de los modelos matemáticos con los modelos estocásticos.

Las investigaciones más significativas fueron realizadas entre las décadas de 1970, 1980 y 1990. En los últimos quince años, a nivel internacional, han sido muy pocos los avances presentados en materia de nuevas metodologías para la administración de la cadena de suministro de sangre; y a nivel nacional, en la revisión del estado de arte, no se encontraron estudios significativos en el desarrollo de modelos para aplicar en bancos de sangre.

Las investigaciones de los últimos años referentes al modelamiento para el control de inventario en los bancos de sangre se han focalizado en desarrollar mejores sistemas de información para facilitar de forma más rápida los resultados de la disponibilidad de las unidades sanguíneas y un mejor conocimiento de la demanda para poder establecer un ordenamiento confiable de los pedidos. Para lo anterior, además de los modelos matemáticos y estocásticos tradicionales, han contribuido algunas técnicas meta-heurísticas como búsqueda tabú y colonia de hormigas, la incorporación de algunas filosofías de mucha visibilidad en la industria como la teoría de restricciones, el análisis de costos, la creación de escenarios, la caracterización de la población donante y la evaluación de las mejores prácticas.

Los modelos matemáticos y estocásticos son una valiosa alternativa en la planeación y control de la cadena de abastecimiento de sangre, y a pesar de que no permiten alcanzar resultados óptimos, permiten un manejo adecuado de la complejidad presente en la interrelación entre variables y parámetros que componen el sistema, lo cual consolida a la investigación multidisciplinaria como una alternativa que permite grandes aportes de la investigación de operaciones y la estadística a un sector administrado tradicionalmente por profesionales de la salud. Los tomadores de decisiones en la red de abastecimiento de sangre siempre deberan balancear el esfuerzo en el desarollo de los modelos para toma de decisones frente a los resultados esperados, los cuales en muchas casos no ofrecen el nivel de confiabilidad requerido.

La utilización de las economías de escala es un argumento a favor de la consolidación de los bancos de sangre en grandes centros regionales, aun cuando faltan soportes experimentales adecuados y los rangos de eficiencia no están relacionados con características demográficas o socioeconómicas del área del Centro Regional de Sangre. En este sentido, el documento contribuye a los esfuerzos investigativos de interés nacional, encaminados a suministrar lineamientos que permitan mejorar el servicio de abastecimiento en los bancos de sangre hospitalarios y su consolidación a través de una gran red regional o Nacional; dentro del marco de la Resolución 167 de enero de 1997 del Ministerio de Salud y la "Política Nacional de Sangre" de 2007.

\section{REFERENCIAS}

Abbot, R. D. (1997). Recycling older blood by integration into the inventory of a single large hospital blood bank, a computer simulation application. Transfusion, 18, 709-715.

Alizadeh, M., Eskandari, H., \& Sajadifar, S. M. (2014). A modified (S-1, S) inventory system for deteriorating items with Poisson demand and non-zero lead-time. Applied Mathematical Modelling, 38, 699-711.

Ballou, R. (2004). Logística, Administración de la cadena de abastecimiento. México: Editorial Prentice hall.

Belien, J., \& Forcé, H. (2012). Supply chain management of blood products: A literature review. European Journal of Operational Research, 217, 1-16.

Bellabdaoui, A., Bennis, I., Saadi, J., \& Benajiba, M. (2014). Optimisation des niveaux de stock dans les centres de transfusion sanguineau Maroc. Transfusion Clinique et Biologique, 21, 15-22.

Blake, J., \& Hardy, M. (2014). A generic modelling framework to evaluate network blood management policies: The Canadian Blood Services experience. Operations Research for Health Care, 3,116-128. 
Brennan, J., Bruce, G., \& Rappoport, H. (1992). Go with the flow: improving red cross blood mobiles using simulation analysis. Interfaces, 22(5), 1-13.

Brodheim, E., \& Prastacos, G. (1979). A regional blood management system with prescheduled deliveries. Transfusion, 19, 455-462.

Broyles, J. R., Cochran, J. K., \& Montgomery, D. C. (2010). A statistical Markov chain approximation of transient hospital inpatient inventory. European Journal of Operational Research, 207, 1645-1657.

Chapman, J. F. (2004). Blood inventory Management. Vox Sanguinis. Londres, 87(2), 143-145.

Cohen, M. (1976). Analysis of single critical number ordering policies for perishable inventories. Operations Research, 24, 726.

Cohen, M., \& Pierskalla, W. (1975). Management policies for a regional blood bank. Transfusion, 15, 58-69.

Dillon, M., Oliveira, F., \& Abbasi, B. (2017). A two-stage stochastic programming model for inventory management in the blood supply chain. International Journal of Production Economics, 187, 27-41.

Duan, Q., \& Liao, T. W. (2014). Optimization of blood supply chain with shortened shelf lives and ABO compatibility. International Journal of Production Economics, 153, 113-129.

Duong, L., Wood, L., \& Wang, W. (2015). A multi-criteria inventory management system for perishable \& substitutable products. Procedia Manufacturing, 2, 66-76.

Elston,R., \& Pickerell, J. (1963). A stastitical approach to ordering and usage policies and usage policies for a hospital blood bank. Transfusion, 3, 43.

Fano, R., \& Longres, A. (1997). Inventario mínimo de componentes sanguíneos en un servicio de hemoterapia de la ciudad de La Habana. Revista Cubana de Medicina Militar, 27(1).
Forrester, J. W. (1973). Industrial dynamics. Cambridge, Massachusetts: MIT press.

Fries, B. (1975). Optimal ordering policy for a perishable commodity with fixed lifetime. Operations research, 23, 46-61.

Gunpinar, S., \& Centeno, G. (2015). Stochastic integer programming models for reducing wastages and shortages of blood products at hospitals. Computers \&Operations Research, 54, 129-141.

Haijema, R., Van Der Wal, J., \& Van Dick, N. (2007). Blood patelet production: Optimization by dinamic programming and simulation. Computers \& Operations Research, 34(3).

Hosseinifard , Z., \& Abbasi, B. (2016). The inventory centralization impacts on sustainability of the blood supply chain. Computers \& Operations Research.

Jagannathan, R., \& Sen, P. (1986). Storingcrossmatched blood. Management Science, (3).

Jagannathan, R., \& Tarun, S. (2005). Storing crossmatched blood: a perishable inventory model with prior allocation. Management Science, 37.

Jennings, J. (1973). Blood bank inventory control. Management Science, 19, 637-645.

Katsaliak, K., \& Brailsfor, S. (2007). Using simulation to improve the blood supply chain. The Journal of the Operational Research Society, 58(2), 219-228.

Ke-ming \& Zu-jun (2015). Age-based policy for blood transshipment during blood Shortage. Transportation Research, 80, 166-183.

Kolesar, P. (1973). Comments on a blood bank model of Pegels \& Jelmert. Operation Research, $21,856-858$.

Kopach, R., Balcioglu, B., \& Carter, M. (2008). Tutorial on constructing a red blood cell inventory management system with two demand rates. European Journal of Operational Research, 185, 1051-1059. 
Kowalski, J. M., France, C. R., Whitehouse, L., \& Himanan, L. K. (2014). Blood donation fears inventory: development and validation of a measure of fear specific to the blood donation setting. Transfusion and Apheresis Science, 51,146-151.

Kozan, E. (2003). A blood bank inventory control model for red blood cells. International Journal of Healthcare Technology and Management, 1, 1-2.

Nahmias, S. (1975). Perishable inventory theory: a review. Operations Research, (6).

Nahmias, S. (1982). Perishable inventory theory: a review. Operations Research, 30, 680-707.

Nahmias, S., \& Pierskalla, W. (1982). Optimal ordering policies for perishable inventory. The institute of management sciences, 2, 485-493.

Osorio, A. F., Brailsford, S., \& Smith, H. (2014). Un modelo de optimización bi-objetivo para la selección de tecnología y asignación de donantes en la cadena de suministro de sangre. Revista S \& T, 12(30), 9-24.

Pegels, C., \& Jelmert, A. (1970). An evaluation of blood inventory policies: a markov chain application. Operation research, 18, 1087.

Pereira, A. (2005). Blood inventory management in the type and screen area. Vox Sanguinis, 89(4), 245-250.

Pierskalla, W. (2004). Supply chain management of blood banks. Operations research and health care: a handbook of methods and applications. ed. kluwer academic publishers, 103-145.

Prastacos, G. (1984). Blood inventory management. Management Science, 16(3).

Prastacos, G. (1998). Blood inventory management: an overview of theory and practice. Management Science, 30(7), 777-800.

Puranam, K., Novak, D., Lucas, M., \& Fung, M. (2017). Managing blood inventory with multiple independent sources of supply. European Journal of Operational Research, 259, 500-511.
Roberts, N., et al. (1983). Introduction to computer simulation - the systems dynamics approach. New York: AdisonWisley publishing company.

Saracoglu, L., Topaloglu, S., \& Keskintur, T. ( 2014). A genetic algorithm approach for multi-product multi-period continuous review inventory models. Expert Systems with Applications, 41, 8189-8202.

Shoukath Ali., K., \& Ramaswany, N. (1991). Simulation of blood bank inventory management systems. Systems dynamics approach, 11-12.

Silva-Filho, O., Cezarino, W., \& Salviano, G. (Mayo, 2012). A Decision-making Tool for Demand Forecasting of Blood Components. Proceedings of the 14th IFAC Symposium on Information Control Problems in Manufacturing, Bucarest, Rumania.

Silva-Filho, O., Carvalho, M., Cezarino, W., Silva, R., \&Salviano, G. (Septiembre, 2013). Demand Forecasting for Blood Components Distribution of a Blood Supply Chain. 6th IFAC Conference on Management and Control of Production and Logistics, Fortaleza, Brazil.

Sirelson, V., \& Brodheim, E. (1991). A computer planning model for blood platelet production and distribution. computer methods and programs. Biomedicine, 35, 279-91.

Van Zyl, G. J. (1964). Inventory control for perishable commodities [Ph. D. thesis]. University of North Carolina, Chapel Hill, N.C.

Vaughan, T. (1988). An analytical model of the blood inventory system at the hospital and regional levels [Doctoral thesis]. Department of Business Administration, University of Iowa, USA.

Velasco, N., \& Amaya, C. (2011). La salud, de la mano de la Ingeniería Industrial. Revista Contacto.

Vidal, C. J. (2010). Fundamentos de gestión de inventarios. Santiago de Cali: Universidad del Valle.

Williams, W. (2000). Hematology. Saint Louis: MC Graw Hill. 